BULL. AUSTRAL. MATH, SOC.

VOL. (1986), 475-476.

\title{
THE SIMPLE INTERSECTION: CAPACITY AND DELAY
}

\author{
Ashley Wayne Plank
}

A simple intersection is the junction of two single-lane road traffic streams, one of which (the major stream) has absolute priority over the other (the minor stream). The problem of determining the capacity of the minor stream and the delay experienced by minor vehicles wishing to merge with or cross the major stream, under steady-state conditions, is apparently a well-researched subject. An important aspect of this problem has however been largely neglected.

Minor vehicles are classified as either consistent or inconsistent, and the population of minor vehicles as either homogeneous or nonhomogeneous. Although considerable attention has been paid the incorporation of inconsistency in models dealing with capacity and/or delay, the nonhomogeneity factor has been comparatively ignored, or dealt with incorrectly. A notable exception is the treatment of delay by Yeo and Weesakul [1]. The assumptions made in their paper however differ in an important respect from the more realistic assumptions of this thesis and part of this work is concerned with comparing the two approaches.

The major contention of this thesis is that inconsistency and nonhomogeneity must be modelled separately. The effect on the performance of the intersection due to inconsistency of drivers and nonhomogeneity across the drivers are in opposite directions. Inconsistency tends to

Received 29 November 1985. Thesis submitted to University of New South Wales, May 1985. Degree approved September 1985. Supervisor: Dr. E.A. Catchpole.

Copyright Clearance Centre, Inc. Serial-fee code: 0004-9727/86 $\$ A 2.00+0.00$. 
increase capacity and reduce delay while nonhomogeneity tends to reduce capacity and increase delay.

The thesis comprises four chapters. Chapter 1 critically reviews the literature on the mathematical modelling of road traffic flow with emphasis on the stochastic description of the headway process. Chapters 2, 3, and 4 deal respectively with the capacity problem, the delay problem for a solitary minor vehicle, and the delay problem for queued minor vehicles. Each of these chapters contains a critical appraisal of the relevant literature, and provides solutions assuming inconsistency and/or nonhomogeneity. The solutions for the nonhomogeneous cases are completely new. Aspects of the solutions provided in the homogeneous cases are new. Extensive use is made of indicator functions and recursive definitions for random variables throughout. Of particular interest are the many formulae derived for capacity and the first two moments of delay to a solitary minor vehicle for specific major-stream headway distributions, notably some based on the assumption of an alternating renewal headway structure. No such simple formulae exist for the queued delay problem. However, iterative formulae are developed for the first two moments of delay for the consistent, homogeneous case, the inconsistent, homogeneous case, and the consistent, nonhomogeneous case. Computer prograns are given in the first and last of these cases.

\section{References}

[1] G.F. Yeo and B. Weesakul, "Delays to road traffic at an intersection", J. App Z. Prob. 1 (1964), 297-310.

School of Information Sciences

Canberra College of Advanced Education

P.O. Box 1

Belconnen, A.C.T. 2616

Australia 